\title{
A INFLUÊNCIA DAS MÍDIAS SOCIAIS NO USO DE MEDICAMENTOS
}

\author{
THE INFLUENCE OF SOCIAL MEDIA ON THE USE OF MEDICINES
}

\section{LA INFLUENCIA DE LAS REDES SOCIALES EN EL USO DE MEDICAMENTOS}

\author{
Fernanda do Nascimento Wolff'; Leyde Daiane de Peder ${ }^{2}$
}

\author{
1 - Centro Universitário da Fundação Assis Gurgacz (FAG) \\ 2 - Professora do curso de Farmácia do Centro Universitário da Fundação Assis Gurgacz
}

\section{RESUMO:}

Com a expansão que a internet vem tomando em conjunto com a visibilidade de pessoas que trabalham com as mídias sociais disponíveis nela, pode ocorrer a influência do uso de produtos e até mesmo medicamentos. A vista disso este estudo abordará como as pessoas são influenciadas digitalmente se tratando do uso de medicamentos, onde em grande parte iniciam o tratamento sem prescrição médica, gerando a automedicação e podendo resultar em efeitos colaterais e reações adversas relacionados a medicamentos. Estudo descritivo e quantitativo, realizado com acadêmicos de um centro universitário do município de Cascavel-PR em março de 2021, utilizando-se instrumento para coleta de dados, após aprovação pelo Comitê de Ética em Pesquisa (CEP). Observou-se que dos 107 acadêmicos entrevistados, $65,42 \%$ realizaram a automedicação em virtude da influência das mídias sociais, onde $68 \%$ eram do curso de Farmácia, sendo o analgésico a classe medicamentosa mais utilizada $(42,99 \%)$ podendo estar relacionado com o motivo pela busca de medicamentos mais citado em $71,96 \%$ das situações a cefaleia. Dos entrevistados, 30,84\% citaram a facilidade de acesso ao medicamento como o maior motivo que levaram a automedicação. A automedicação é bastante comum entre os acadêmicos, demonstrando maior frequência nos acadêmicos que cursam Farmácia.

DESCRITORES: Automedicação. Efeitos Colaterais e Reações Adversas Relacionados a Medicamentos. Mídias Sociais.

\section{ABSTRACT:}

With the expansion that the internet has been taking in conjunction with the visibility of people who work with the social media available on it, there may be an influence of the use of products and even medicines. In view of this, this study will address how people are digitally influenced when it comes to the use of medications, where in large part they start treatment without a prescription, generating self- medication and may result in side effects and adverse reactions related to medications. Descriptive and quantitative study, carried out with academics from a university center in the municipality of Cascavel-PR in March 2021, using an instrument for data collection, after approval by the Research Ethics Committee. It was observed that of the 107 academics interviewed, $65.42 \%$ underwent self-medication due to the influence of social media, where $68 \%$ were from the Pharmacy course, with analgesics being the most used medication class $(42.99 \%$,) which may be related to the reason for seeking medication most cited in $71.96 \%$ of headache situations. Of the interviewees, $30.84 \%$ cited the ease of access to the medication as the main reason that led to self-medication. Self-medication is quite common among academics, showing greater frequency in academics who study Pharmacy. 
DESCRIPTORS: Self-medication. Drug-Related Side Effects and Adverse Reactions. Social media.

\section{RESUMEN:}

Con la expansión que ha venido tomando internet en conjunto con la visibilidad de las personas que trabajan con las redes sociales disponibles en ella, puede haber influencia del uso de productos e incluso medicamentos. Ante esto, este estudio abordará cómo las personas son influenciadas digitalmente en el uso de medicamentos, donde en gran parte inician tratamiento sin prescripción médica, generando automedicación y pueden resultar en efectos secundarios y reacciones adversas relacionadas con los medicamentos. Estudio descriptivo y cuantitativo, realizado con académicos de un centro universitario del municipio de Cascavel-PR en marzo de 2021, utilizando un instrumento de recolección de datos, previa aprobación del Comité de Ética en Investigación. Se observó que de los 107 académicos entrevistados, el 65,42\% se automedicaron por influencia de las redes sociales, donde el $68 \%$ eran del curso de Farmacia, siendo los analgésicos la clase de medicación más utilizada $(42,99 \%$,) lo que puede estar relacionado al motivo de búsqueda de medicación más citado en el $71,96 \%$ de las situaciones de cefalea. De los entrevistados, el $30,84 \%$ citó la facilidad de acceso a la medicación como el principal motivo que llevó a la automedicación. La automedicación es bastante común entre los académicos, mostrando una mayor frecuencia en los académicos que estudian Farmacia.

DESCRIPTORES: Automedicación. Efectos secundarios y reacciones adversas relacionados con los medicamentos. Redes sociales.

\section{INTRODUÇÃO}

Atualmente a internet é utilizada para uma infinidade de atividades, como nas relações econômicas, na política, na cultura e na saúde. Tudo está envolto nas chamadas mídias sociais. Mesmo com sua curta história, as mídias sociais na Internet, têm mantido um papel cada vez mais relevante na vida dos jovens (CARDOSO, 2011).

Com a expansão que a internet vem tomando em conjunto com a visibilidade de pessoas que trabalham diretamente com as mídias sociais disponíveis nela, pode ocorrer a influência direta e/ou indireta do uso de produtos e até mesmo medicamentos. Estes podem ser medicamentos isentos de prescrição médica e até mesmo medicamentos tarjados, isto é, aqueles que somente podem ser vendidos por meio de uma receita médica. Mesmo que as propagandas para com esses medicamentos fiquem restritas aos meios de comunicação dirigidos, destinados exclusivamente aos profissionais de saúde habilitados, essa prática pode ser observada em meios de comunicação (FERNANDES, CEMBRANELLI, 2014) (NASCIMENTO, 2007).

Pelo fato de muitas pessoas acompanharem e confiarem nos influenciadores digitais, acabam adquirindo e iniciando um tratamento com produtos e/ou medicamentos indicados por eles, sem se preocupar em procurar acompanhamento de um profissional 
qualificado, e sem ter um mínimo de conhecimento sobre os riscos que os mesmos podem acarretar (CORREIA et al, 2019). Desta forma, induzindo uma prática de autocuidado ou de automedicação, além de resultar em grandes problemas familiares, como a rebeldia através da internet e o vício que este meio pode causar nos jovens. A vista disso, as mídias sociais, pelo fato de serem de fácil acesso em sua maioria, podem aproximar as relações entre as pessoas, gerando uma transmissão rápida de informações, como por exemplo, a indicação de um medicamento pela influência de um "digital influencer" ou de um amigo (NARDI; TERRA, 2014).

Ainda que a prática da automedicação resulte em consequências prejudiciais às pessoas que a adotam, agravando muitas vezes a qualidade de vida das mesmas a longo prazo, essa é uma prática vista frequentemente no ambiente acadêmico, tanto pelo fato de não conhecer os efeitos colaterais, quanto pela facilidade em encontrar informações por meio do ambiente virtual, em que se encontram vários tratamentos para os sintomas existentes (COELHO et al, 2016).

Com isso, sabe-se que a dispensação de medicamentos com "tarja vermelha" deveria ocorrer somente com a apresentação de uma prescrição médica, porém, não é sempre que durante a aquisição de alguns medicamentos indicados pelos influenciadores digitais, a apresentação da receita médica é realizada. Ação esta, que além da prática da automedicação, pode acabar resultando no uso irracional de medicamentos, onde se tem um consumo excessivo dos mesmos, por não ter nenhum controle ou informação prestada por um profissional de saúde. Essas situações podem causar malefícios à saúde de modo geral, tais como: efeitos adversos, eficácia limitada, insucesso no tratamento dos sintomas, resistência a medicamentos, interações medicamentosas e a farmacodependência (CARDOSO, 2011).

Sendo assim, o presente estudo se torna importante para elucidar a automedicação realizada a partir da influência de mídias sociais pode acarretar e para mostrar o quanto esses jovens são susceptíveis a esta prática.

\section{METODOLOGIA}

Trata-se de um estudo descritivo de abordagem quantitativa, desenvolvido em um centro universitário do município de Cascavel no estado do Paraná, Brasil, com estudantes de cinco cursos nas áreas de gestão, saúde e exatas. Com o objetivo de abranger diferentes áreas de atuação. 
A população amostra foi constituída por aqueles acadêmicos que aceitaram participar da pesquisa respondendo um formulário on-line e que preencheram o Termo de Consentimento Livre e Esclarecido (TCLE). Os seguintes critérios de inclusão os participantes deveriam possuir, idade igual ou superior a 18 anos, e ser acadêmico dos cursos de Farmácia, Enfermagem, Administração, Engenharia Civil e Arquitetura de um Centro Universitário do município de Cascavel-PR. Foram excluídos do estudo aqueles que tinham idade inferior a 18 anos, e que estivessem cursando outros cursos.

De acordo com a faixa etária estabelecida, foi enviado via on-line através de um aplicativo de mensagens, um questionário estruturado auto aplicativo a 470 alunos dos cursos a cima, obtendo reposta de 107 alunos que aceitaram participar da pesquisa.

O questionário foi desenvolvido pela pesquisadora e constituído por 14 questões objetivas e subjetivas, onde foram levantados os dados pessoais, como idade e sexo, influência do uso de medicamentos, a mídia social que o influencia, uso de medicamentos sem prescrição médica, classe do medicamento utilizado, efeito adverso ou alergia ao usar um medicamento sem prescrição, lê a bula, tem conhecimento dos possíveis riscos da automedicação, conhece a definição de automedicação, sintomas que busca amenizar e fatores para realizar a automedicação.

Ressalta-se que todas as etapas analíticas, bem como toda a análise estatística descritiva, foram procedidas da síntese das informações em um banco de dados eletrônico do software Microsoft Office Excel 2016, os quais foram apresentados em tabelas e figuras para melhor visualização.

O projeto seguiu todos os preceitos éticos e foi aprovado em 26/03/2021 pelo Comitê de Ética em Pesquisa (CEP) do Centro Universitário Assis Gurgacz, sob o parecer no 4.615.246 (CAAE: 40039220.6.0000.5219).

\section{RESULTADOS}

Foram entrevistados 107 estudantes divididos entre cinco cursos de três diferentes áreas (gestão, saúde, exatas). Do total de entrevistados, 76 (71,02\%) eram da área da saúde, $83(77,57 \%)$ eram do sexo feminino, quanto à idade, a mesma variou de 18 a mais de 50 anos.

Em relação à automedicação em virtude da influência das mídias sociais, 70 $(65,42 \%)$ afirmaram que já realizaram essa prática, 102 (95,32\%) afirmaram conhecer a definição de automedicação e 36 (33,64\%) não tem conhecimento dos possíveis riscos da 
automedicação. De todas as pessoas que alegaram que se automedicaram por influência das mídias sociais 11 (10,28\%) relataram ter apresentado algum efeito adverso ou alergia, sendo que 2 relataram ter apresentado tontura, 3 relataram ter desenvolvido alergias cutâneas, 2 sofreram um choque anafilático, além das demais reações adversas ao medicamento.

A Tabela 1 apresenta os resultados dos testes de associação entre a variável automedicação, o perfil dos entrevistados e outras questões relacionadas à saúde.

Tabela 1 - Resultados dos testes de associação entre automedicação praticada pelos acadêmicos. Cascavel, PR, Brasil, 2021

\begin{tabular}{lcc}
\hline Variáveis & Total (\%) & Automedicação (\%) \\
\hline Sexo & $83(77,57)$ & 57 \\
Feminino & $24(22,42)$ & 8,41 \\
Masculino & & \\
Idade (anos) & $93(83,91)$ & 57,94 \\
18 a 30 & $11(10,28)$ & 7,47 \\
31 a 50 & $(2,8)$ & 0 \\
Mais de 50 & & 47,7 \\
Uso sem prescrição médica & $79(73,83)$ & 17,75 \\
Sim & $28(26,16)$ & 14,95 \\
Não & & 35,51 \\
Lê a bula & $26(24,29)$ & \\
Sim & $28(26,16)$ & \\
Não & $53,53)$ & \\
Poucas vezes & & \\
\hline
\end{tabular}

Com relação à classe dos medicamentos utilizados por influência, 5 pessoas $(4,67 \%)$ disseram buscar por medicamentos para emagrecer, 3 (2,8 \%) afirmaram utilizar 
medicamentos para tratar problemas capilares e $2(1,86 \%)$ pessoas disseram utilizar medicamentos para dormir.

Os problemas de saúde cujo o tratamento foi realizado por automedicação estão apresentados na Tabela 2, juntamente com os motivos para a prática.

Tabela 2 - Influência da prática da automedicação: Motivos pela busca de medicamentos, e os motivos para prática. Cascavel, PR, Brasil, 2021

Variáveis

n (\%)

\section{Motivos pela busca de medicamentos}

Dor muscular $55(51,4)$

Cefaleia

$77(71,96)$

Cólica

$39(36,44)$

Cravos e espinhas

$20(18,69)$

Tosse

$25(23,36)$

Distúrbios gastrintestinais

$22(20,56)$

Gripe ou resfriado

$59(55,14)$

Alergias

$39(36,44)$

Outros

$7(6,54)$

\section{Motivos para a prática}

Dificuldade de acesso aos serviços de saúde

$14(13,08)$

Facilidade de acesso ao medicamento

$33(30,84)$

Aversão ao ambiente hospitalar

$5(4,67)$

Por ter experiência com o medicamento

$31(28,97)$

Por confiar na indicação

$16(14,95)$

Outros

$2(1,86)$

As classes medicamentosas mais utilizadas entre os acadêmicos estão demonstradas na Tabela 3. 
Tabela 3 - Frequência das classes de medicamentos consumidas pelos acadêmicos de um centro universitário do município de Cascavel no estado do Paraná, Brasil, 2021

\begin{tabular}{lcc}
\hline Classe de medicamentos & Absoluto (relativo) & Valor p \\
\hline Analgésicos & $46(42,99)$ & $<0,0001$ \\
Antibióticos & $1(0,93)$ & \\
Antivirais & $16(14,95)$ & \\
Anti-inflamatórios & $24(22,42)$ & \\
Outros & $20(18,7)$ & \\
\hline
\end{tabular}

Quando comparada a área da saúde com as demais, em relação às influências relatadas para a prática da automedicação, houve uma associação significativa, conforme apresentado na Figura 1.

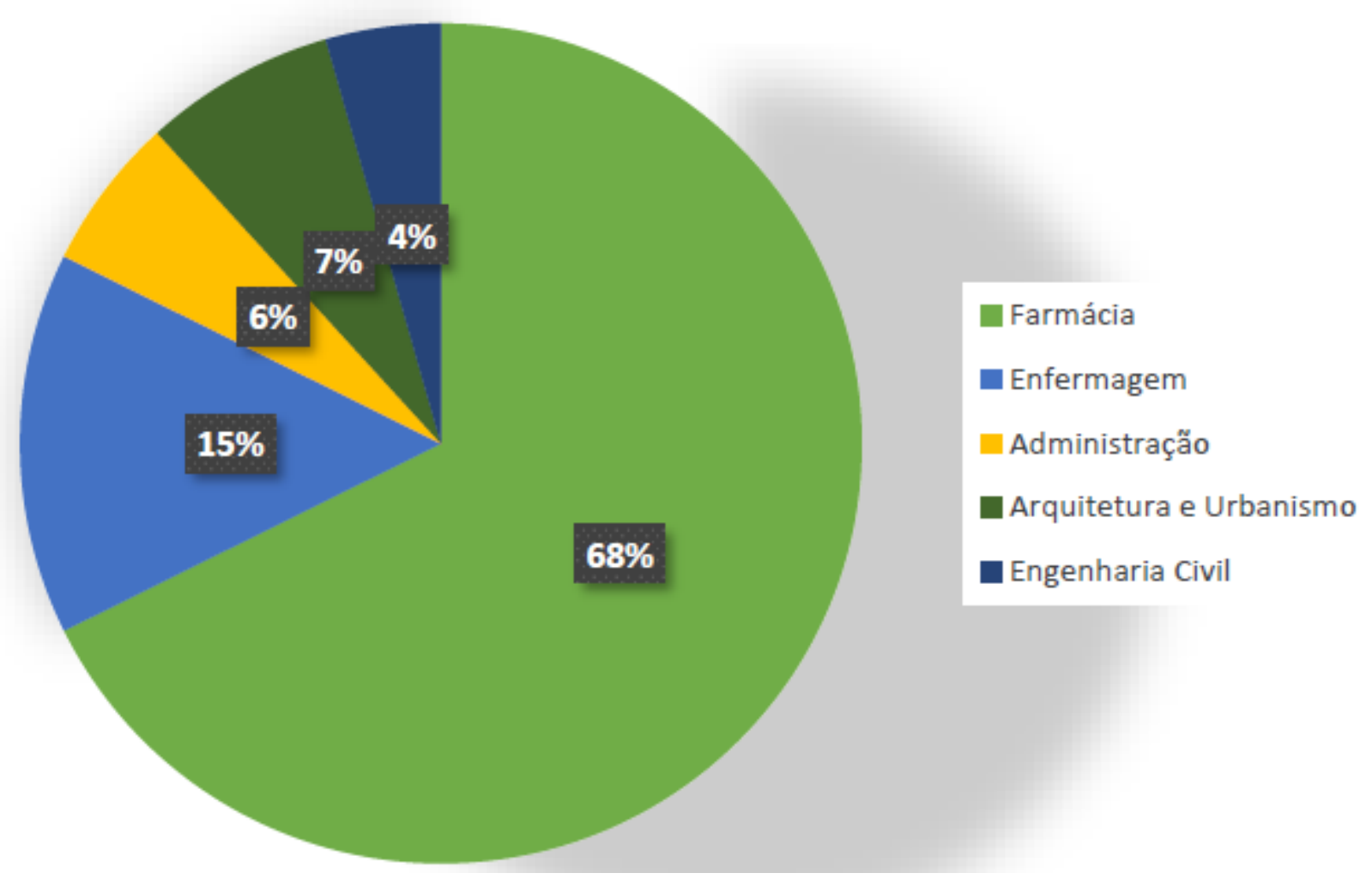

Figura 1 - Comparação entre as áreas de formação com as influências relatadas para a prática da automedicação. $p<0,0001$. Cascavel, PR, Brasil, 2021 
Quando questionados em relação à mídia social onde foi influenciado a usar um medicamento, os acadêmicos apresentaram respostas diferentes e estas estão compiladas em Figura 2.

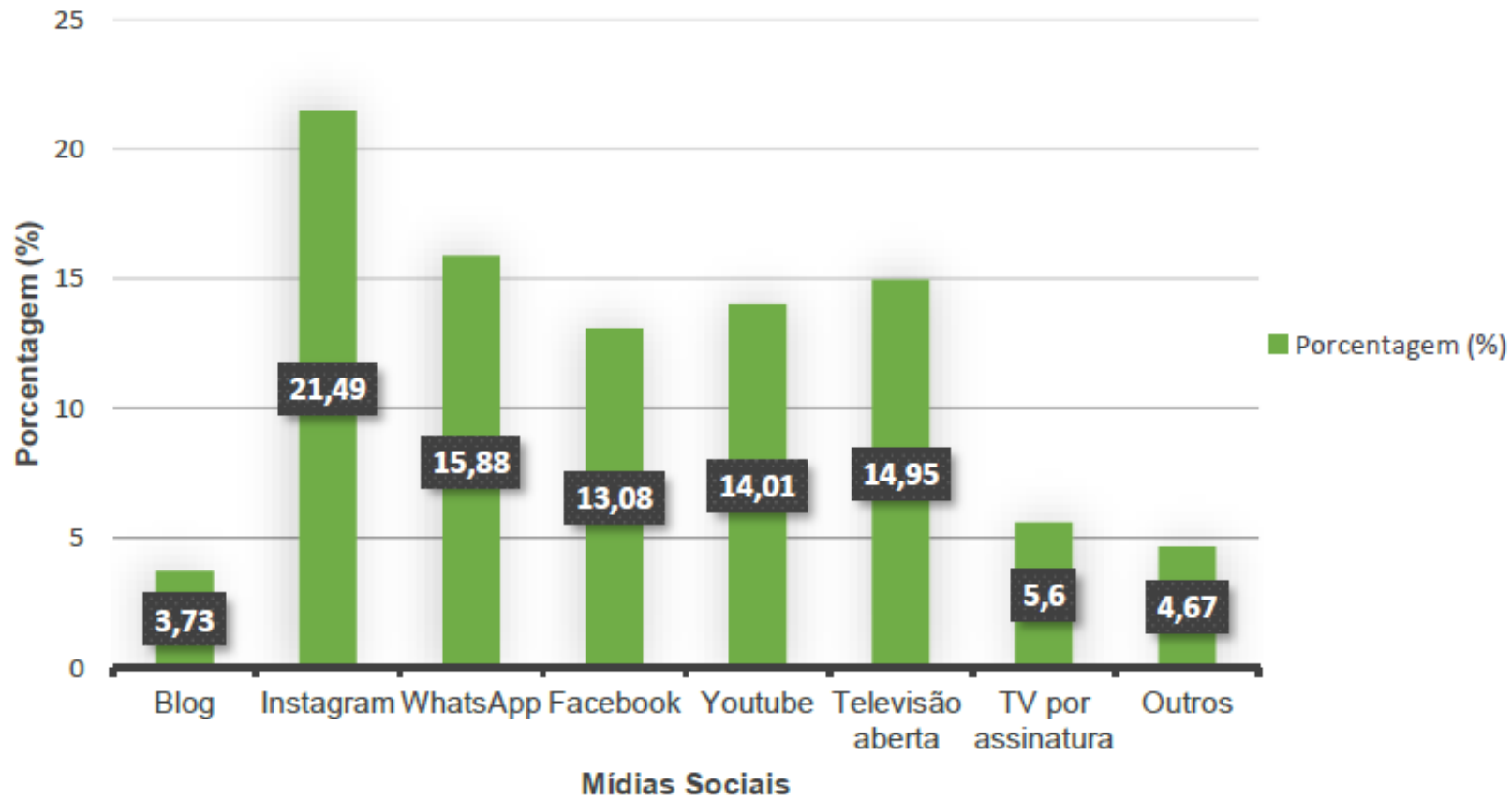

Figura 2 - Porcentagem relacionada a mídia social que influenciou na prática automedicação. Cascavel, PR, Brasil, 2021

\section{DISCUSSÃO}

A automedicação é uma prática muito comum entre todas as faixas etárias, mas o principal foco deste estudo foram os acadêmicos de um Centro Universitário do município de Cascavel no estado do Paraná, os quais em sua maioria são jovens entre 18 e 30 anos, sendo que 57,94\% deles realizaram a automedicação por influência de mídias sociais.

Pelo fato de serem jovens, em geral mantém maior contato com a internet, afirmação essa, que ganha maior relevância quando aliada a dados semelhantes encontrados em um estudo realizado sobre a influência dos meios de comunicação (mídiainternet) na construção de subjetividades de jovens universitários, com idade entre 18 e 23 anos, o qual demostra que $85 \%$ são usuários destes meios de comunicação (CASTANHO, ZORZIM, 2017).

A automedicação é uma conduta comum no Brasil e pode ser definida como uma forma de autocuidado, sendo importante que seja avaliado se a mesma é realizada de 
forma responsável, isto significa afirmar que deve ser realizada no contexto do uso racional de medicamentos. Sendo assim, se torna importante que os acadêmicos utilizem os medicamentos para as suas queixas clínicas, que devem ser caracterizadas no contexto da automedicação como transtornos menores, ou seja, enfermidades de baixa gravidade (ARRAYS, 2002).

Quando considerado o sexo dos participantes, o grupo maioritário foi o das mulheres, sendo que 57\% relataram praticar automedicação, enquanto $8,41 \%$ que afirmaram realizar a prática eram do sexo masculino. Segundo a Revista da saúde pública (2016), a prevalência de automedicação na população brasileira foi maior no sexo feminino, na faixa etária entre 20 e 39 anos.

Em relação à utilização de medicamentos sem apresentação de prescrição médica, das 107 pessoas que participaram desta pesquisa 79 afirmaram utilizar medicamentos sem a prescrição médica. Dado este, que se pode encontrar semelhança quando observado um estudo realizado na zona urbana da cidade de Teresinha-PI no ano de 2011, onde se observou que mais de $92 \%$ das pessoas usavam medicamentos sem a prescrição de um profissional habilitado (MARTINS et al, 2011).

Na presente pesquisa foi observado que os motivos pela busca de medicamentos mais citados para o manejo com automedicação foi a cefaleia em $71,96 \%$ das situações, seguido de gripe e resfriado com $55,14 \%$ e dor muscular em $51,40 \%$ dos relatos. Estes motivos pela busca de medicamentos também podem ser observados em outras pesquisas como os mais comuns para a prática da automedicação (DAMASCENO et al, 2007) (PENNA et al, 2004). Quando investigados os medicamentos selecionados para a automedicação observou-se que entre os mais utilizados estão os medicamentos da classe dos analgésicos com 42,99\% (46) dos casos e Anti-inflamatórios com 22,42\% (24). Estes resultados são coerentes com os principais motivos pela busca de medicamento, sendo a dor de cabeça, onde encontram-se informações em outras pesquisas descritos na literatura (GALATO et al, 2012).

A partir destes dados, não se deve desconsiderar que a automedicação pode acarretar consequências prejudiciais à saúde como riscos de interações medicamentosas, reações adversas, insucesso no tratamento dos sintomas, resistência a medicamentos, e farmacodependência (BAGGIO, FORMAGGIO, 2009).

Entre os motivos que levaram a automedicação os mais citados pelos acadêmicos foram a facilidade de acesso ao medicamento $(30,84 \%)$ e por ter experiência com o medicamento $(28,97 \%)$. Sendo este resultado semelhante ao encontrado por Mussolin em 
um estudo realizado entre universitários de relações públicas e enfermagem, onde destaca essa facilidade como um fator importante para a prática em questão (MUSSOLIN, 2004).

No curso de Farmácia, a incidência da automedicação foi significativamente maior (68\%) em relação aos outros cursos. Incidência essa, que pode ser justificada pelo fato de os estudantes de Farmácia abranger a maior parte da população amostra, sendo estes os que mais responderam o questionário para o estudo em questão.

Com isso, observa-se um estudo semelhante realizado por Lima, com acadêmicos do curso de farmácia em uma instituição privada de ensino superior em Fortaleza-CE. Onde o resultado afirmou realizarem a prática da automedicação 99,51\% dos acadêmicos, dado este, que pode ser justificado em virtude de os participantes serem estudantes do curso de Farmácia, dispondo de uma maior confiança e segurança em relação aos medicamentos, já que possuem grande conhecimento sobre os mesmos, assim como sua indicação, posologia e os efeitos adversos. O número de automedicações nesses universitários pode estar relacionado com o período da graduação, como também ao conjunto de conhecimentos obtidos ao longo do curso, tornando-os assim, mais confiantes com suas próprias habilidades técnicas e teóricas. Logo, esperasse que esses conhecimentos sejam determinantes para atitudes conscientes e adequadas a sua graduação (LIMA et al, 2018).

Diante das informações coletadas em relação a mídia social onde foi influenciado a usar um medicamento, boa parte dos acadêmicos indicou ter sido mais influenciado através da mídia social Instagram (21,49\%) e pelo aplicativo de mensagens WhatsApp $(15,88 \%)$. Dados estes, que podem ser amparados pelo estudo de Melo sobre a automedicação e uso indiscriminado de medicamentos durante a pandemia do novo Coronavírus (COVID-19). Estudo este, que fala sobre a prescrição e o uso de medicamentos que não tenham sido aprovados pelas autoridades reguladoras, para tratar ou prevenir o COVID-19, onde recebeu grande visibilidade quando o "tratamento precoce" e o "kit-covid" foram divulgados e o seu uso incentivado amplamente nas mídias sociais (WhatsApp, Facebook e Instagram) por profissionais médicos (MELO et al, 2021).

Se torna fundamental incentivar os profissionais da área de saúde, para que possam trabalhar a conscientização entre a população, com campanhas realizadas nas próprias mídias sociais, de modo a reduzir a prática de automedicação, por meio de educação em saúde da comunidade. Orientando quanto ao uso racional de medicamentos, abordando os riscos que os mesmos podem gerar e complicações que a automedicação apresenta em geral. 
O estudo em questão dispõe de algumas limitações, como o fato de durante a coleta de dados as aulas estavam sendo realizadas remotamente, devido a pandemia do COVID19 , que impediu que a coleta ocorresse pessoalmente, fato que poderia aumentar o tamanho da amostra em questão. Amostra esta, que teve seu tamanho reduzido, no entanto, não foi um impedimento, pelo contrário, pode ser de grande importância, a partir do momento em que os resultados podem auxiliar na constatação da automedicação que pode ser danosa à saúde, trazendo prejuízos a longo prazo.

\section{CONCLUSÃO}

Este estudo mostrou que a automedicação é bastante comum entre os acadêmicos, demonstrando uma frequência significativamente maior nos acadêmicos que cursam Farmácia. Observa-se que essa prática se torna mais comum, principalmente pela facilidade de acesso aos medicamentos. Nota-se também que a classes de medicamentos mais utilizadas foram analgésicos e anti-inflamatórios, por um número estatisticamente maior de pessoas. Mesmo que a utilização de medicamentos sem prescrição possa parecer tão normal quanto comum, é necessário muito cuidado, pois pode gerar consequências prejudiciais à saúde.

\section{REFERÊNCIAS}

ARRAYS PSD. O uso irracional de medicamentos e a farmacovigilância no Brasil. Cad. Saúde Pública, Rio de Janeiro. [Internet] 2002; [acesso em 03 mai 2021]. Disponível: https://revista.univap.br/index.php/revistaunivap/article/view/265.

BAGGIO MA, FORMAGGIO FM. Automedicação: desvelando o descuidado de si dos profissionais de enfermagem. [Internet] 2009; [acesso em 03 mai 2021]. Disponível: https://docplayer.com.br/8293704-Automedicacao-desvelando-o- descuidado-de-si.html.

CARDOSO, ACS. As redes sociais online, os jovens e a cidadania. [Internet] 2011; [acesso em 22 out 2020]. Disponível: https://repositorio.iscte- iul.pt/handle/10071/3463.

CASTANHO MIS, ZORZIM TJI. Internet, cultura do consumo e subjetividade de jovens. [Internet] 2017; [acesso em 30 abr 2021]. Disponível: http://www.seer.ufsj.edu.br/index.php 
/revista_ppp/article/view/1904/1277.

COELHO MT, Santos VP, Carmo MB, Souza AC, França CP. Relação entre a Autopercepção do Estado de Saúde e a Automedicação entre Estudantes Universitários. Universidade Federal da Bahia. [Internet] 2016; [acesso em 28 set 2020]. Disponível: https://www5.bahiana.edu.br/index.php/psicologia/article/view/1141/817.

CORREIA CB, TRINDADE KJ, ALMEIDA BA. Fatores Correlacionados à Automedicação entre os Jovens e Adultos uma Revisão Integrativa da Literatura. [Internet] 2019; [acesso em 31 ago 2020]. Disponível: https://revistasfacesa.senaaires.com.br/index.php/iniciacaocientifica/article/view/143/98.

DAMASCENO DD, TERRA FS, ZANETTI HHV, D'ANDRÉA ED. Automedicação entre graduandos de enfermagem, farmácia e odontologia da universidade federal de alfenas. [Internet] 2007; [acesso em 03 mai 2021]. Disponível:https://cdn.publisher.gn1.link/reme.org.br/ pdf/v11n1a08.pdf.

FERNANDES SW, CEMBRANELLI CJ. Automedicação e o Uso Irracional de Medicamentos: O papel do profissional farmacêutico no combate a essas práticas. [Internet] 2014; [acesso em 01 set 2020]. Disponível: https://revista.univap.br/index.php/revistaunivap/ article/view/265/259.

GALATO D, MADALENA J, PEREIRA GB. Automedicação em estudantes universitários: a influência da área de formação. [Internet] 2012; [acesso em 03 mai 2021]. Disponível: https://www.scielosp.org/article/csc/2012.v17n12/3323-3330/pt/.

LIMA DM, SILVA JS, VASCONCELOS LF. Avaliação da prática da automedicação em acadêmicos do curso de farmácia em uma instituição privada de ensino superior em Fortaleza-CE. [Internet] 2018; [acesso em 03 mai 2021]. Disponível: http://publicacoesacademicas.unicatolicaquixada.edu.br/index.php/recsaude/articl e/view/ 2122/pdf.

MARTINS MCC, LEAL LMM, FILHO MDS, COSTA EM, HOLANDA LGM. Uso de medicamentos sem prescrição médica em Teresina, PI. [Internet] 2011; [acesso em 02 mai 
2021]. Disponível: https://periodicos.uninove.br/saude/article/view/2516/1893.

MELO JRR, DUARTE EC, MORAES MV. Automedicação e uso indiscriminado de medicamentos durante a pandemia da COVID-19. [Internet] 2021; [acesso em 03 mai 2021]. Disponível: https://scielosp.org/pdf/csp/2021.v37n4/e00053221/pt.

MUSSOLIN NM. A automedicação: um estudo entre universitários de enfermagem e de relações públicas. [Internet] 2004; [acesso em 03 mai 2021]. Disponível: http://bases.bireme.br/cgi-bin/wxislind.exe/iah/online/?IsisScript=iah/iah.xis\&src=google \&base=LILACS\&lan $g=p \&$ nextAction=Ink\&exprSearch=398677\&indexSearch=ID .

NARDI, VA, TERRA, AE. A influência do entretenimento no comportamento dos jovens. [Internet] 2014; [acesso em 22 out 2020]. Disponível: https://www.redeicm.org.br/revista/ wp-content/uploads/sites/36/2019/06/a2_entretenimento.pdf.

NASCIMENTO, Á. Propaganda de Medicamentos: Como Conciliar Uso Racional e a Permanente Necessidade de Expandir Mercado?. [Internet] 2007; [acesso em 23 jun 2021]. Disponível: https://www.scielo.br/j/tes/a/SsnvSKFP9y9DkV3sHyfj4Wy/?lang=pt.

PENNA AB, BORGES CC, BATISTA RD. Análise da Prática da Automedicação em Universitários do Campus Magnus - Unipac - Barbacena, MG. [Internet] 2004; [acesso em 03 mai 2021]. Disponível: https://www.ufmg.br/congrext/Saude/Saude20.pdf.

Revista de Saúde Pública. Prevalência da automedicação no Brasil e fatores associados. [Internet] 2016; [acesso em 30 abr 2021]. Disponível: https://www.scielosp.org/article/rsp/ 2016.v50suppl2/13s/pt/.

\section{Autor para correspondência:}

Fernanda do Nascimento Wolff

E-mail: fernwolff1997@hotmail.com | Endereço: Rua Prof. Orozendo C. de Jesus 337, 14 de novembro, Cascavel/PR | Telefone: (045) 99807-8202.

Recebido: 06/06/2021 Aceite: 10/08/2021 